Int. J. Advance Soft Compu. Appl, Vol. 13, No. 3, November 2021

Print ISSN: 2710-1274, Online ISSN: 2074-8523

Copyright (C) Al-Zaytoonah University of Jordan (ZUJ)

\title{
An Intelligent Ear Recognition Technique
}

\author{
Yahya Abdulmunem Hussein, ALI Mohammed Sahan, and Ali Sami Azeez \\ Al-Itbi
}

Informatics Dept, Technical College of Management, Middle Technical

University, Baghdad, Iraq

e mail: dralimohammed2@gmail.com and ali.sami@mtu.edu.iq

\begin{abstract}
The human ear has unique and attractive details; therefore, human ear recognition is one of the most important fields in the biometric domains. In this work, we proposed an efficient and intelligent ear recognition technique based on particle swarm optimization, discrete wavelet transform, and fuzzy neural network. Discrete wavelet transform is used to provide comprise and effective features about the ear image, while the particle swarm optimization utilized to select more effective and attractive features. Furthermore, using particle swarm optimization leads to reduce the complexity of the classification stage since it reduces the number of the features. Fuzzy neural network used in the classification stage in order to provide strong distinguishing between the testing and training ear images. many experiments performed using two ear databases to examine the accuracy of the proposed technique. The analysis of the results refers that the presented technique gained high recognition accuracy using various data sets with less complexity.
\end{abstract}

Keywords: Ear recognition; bio-metric; discrete wavelet transform, particle swarm optimization, fuzzy neural network.

\section{Introduction}

Biometrics is a crucial specialty that amalgamates both computer and biological sciences [1,2]. In recent decades biometrics has become one of the most critical research areas and has become a major way of recognition, identification, verification, and authentication [3,4]. Because of its uniqueness and distinguishing traits, biometrics used to measured and analyzed the physiological, behavioral characteristics or a combination of both of these features, such as ear, voice, faces, 
palm veins, fingerprints, dental records, deoxyribonucleic acid, iris, mouse and keystroke dynamics, human gait, etc [5,6]. These unique features afford a more powerful, secure, and reliable than the other traditional features used to identity cards, keys, tokens, and passwords [7-10]. The ears are paired organs; on each side of the head; there is one ear. The ear contains three parts, namely: the outer ear, the middle ear, and the inner ear [11,12]. The visible part is the outer ear which contains the external acoustic meatus and pinna or auricle. The pinna shape of any individual is unlike others. It is used as an identification parameter. The pinna's shape varies according to the ethnic group, race, sex, and age; therefore, the features included in the pinna area considered as a biometric feature [13]. The ear structure is comprise worthy features as well as it changes slightly with age [14]. The outer ear also differs from other biometrics, such as the face, since the face can be affected by facial expression, ageing, wearing sunglasses, makeup effects, and growing or shaving beard [15]. Furthermore, the ear size is bigger compared to the size of iris, retina, and fingerprint, so it is likewise readily capturing from a distance $[16,17]$. The outer ear and its important features have been described by several researchers since it has a significant role in the identification of victims of crimes and criminals or accidents surveillance video, and authentications [18,19]. In 2001, many robberies have happened at gas stations in Utrecht city, Netherlands, biometrics system based on ear helps police for identifying a suspect [20]. In 2013, Biometric systems played essential roles to determine the twosome suspects of the explosions of Boston [21]. In 2006, the ear proposed as a biometric, and the dataset used for tests includes 415 persons, each with images were taken on at least two different dates. The Methods considered involving a Principal component analysis (PCA) technique with 2D intensity images. The system has does a high recognition rate of $97.6 \%$. The outcomes propose a powerful potential for 3D ear shape as a biometric [22]. The researchers in [23] proposed a distinguishing biometric method based on ear, it deals with 2D as well as 3D data employing precomputed voxel closest neighbors approach. The work in [24] presented a rotation invariants ear recognition technique as well as it invariant to both translation and scaling. The accuracy of the experimental results shows almost 98\%. Celia Cintas et.al.[25] presented a strong method using geometric morphometric and deep learning to represent a new automated system for ear detection and extraction of features. A set of manually landmarked examples have been used to training the convolutional neural network $(\mathrm{CNN})$ on it, the trained $\mathrm{CNN}$ has the ability to accurately locate the semi-landmarks and the landmarks with truly comparable by the supervised land marking. In [26] a new human ear identification technique has been proposed based on combining the hierarchical deep features. Examinations are carried on several public datasets: USTB I, II and IIT Delhi I, II. In 2019, an ear recognition method has been suggested based on the speeded-up robust feature (SURF) computer vision algorithm [27]. The SURF algorithm creates a descriptor for matching to detected interest points calculating the similarity measure as the ratio between the numbers of matching interest points within a couple of images to the entire number of 
interest points in the input image. Particle swarm optimization (PSO) algorithm has utilized to improve the performance of this method, by excluding each value in the descriptors either that have negative or it does not influence the similarity measurements. The performance was significantly improved, via improving the execution time plus the recognition accuracy. The execution time was improved by the reduction of the descriptor size, while the accuracy has been improved by the elimination of the values that have a negative influence on the similitude measurements.

The rest of the paper has been organized as follows. The proposed technique reviewed in Section 2. Preprocessing and its techniques went throw in this Section 3. Section 4 goes along with the feature extraction, while PSO discussed in section 5. Fuzzy neural network reviewed in section 6. Details of experimental results is presented in Section 7. The conclusions presented in Section 8.

\section{The Proposed Method}

In this paper an intelligent and accurate ear recognition technique has been proposed using discrete wavelet transform (DWT), PSO, and Fuzzy neural network. The following stages summarized the proposed technique:

1. Preprocessing stage: the purpose of this stage is to enhance the ear image and remove the noise as well as extract the region of interest.

2. Feature extraction stage: the aim of this stage is to provide the ear image's features. The DWT has been adopted provide an accurate representation of ear image with less number of coefficients.

3. Feature selection stage: the purpose of this stage is to select distinct features which lead to reduce the complexity of classification stage and improve the proposed technique. The PSO technique was utilized for this purpose.

4. Classification stage: in this stage the test ear image is classified into recognized or unrecognized. The fussy neural network has been utilized in the classification stage. Figure1 illustrates the proposed technique. 


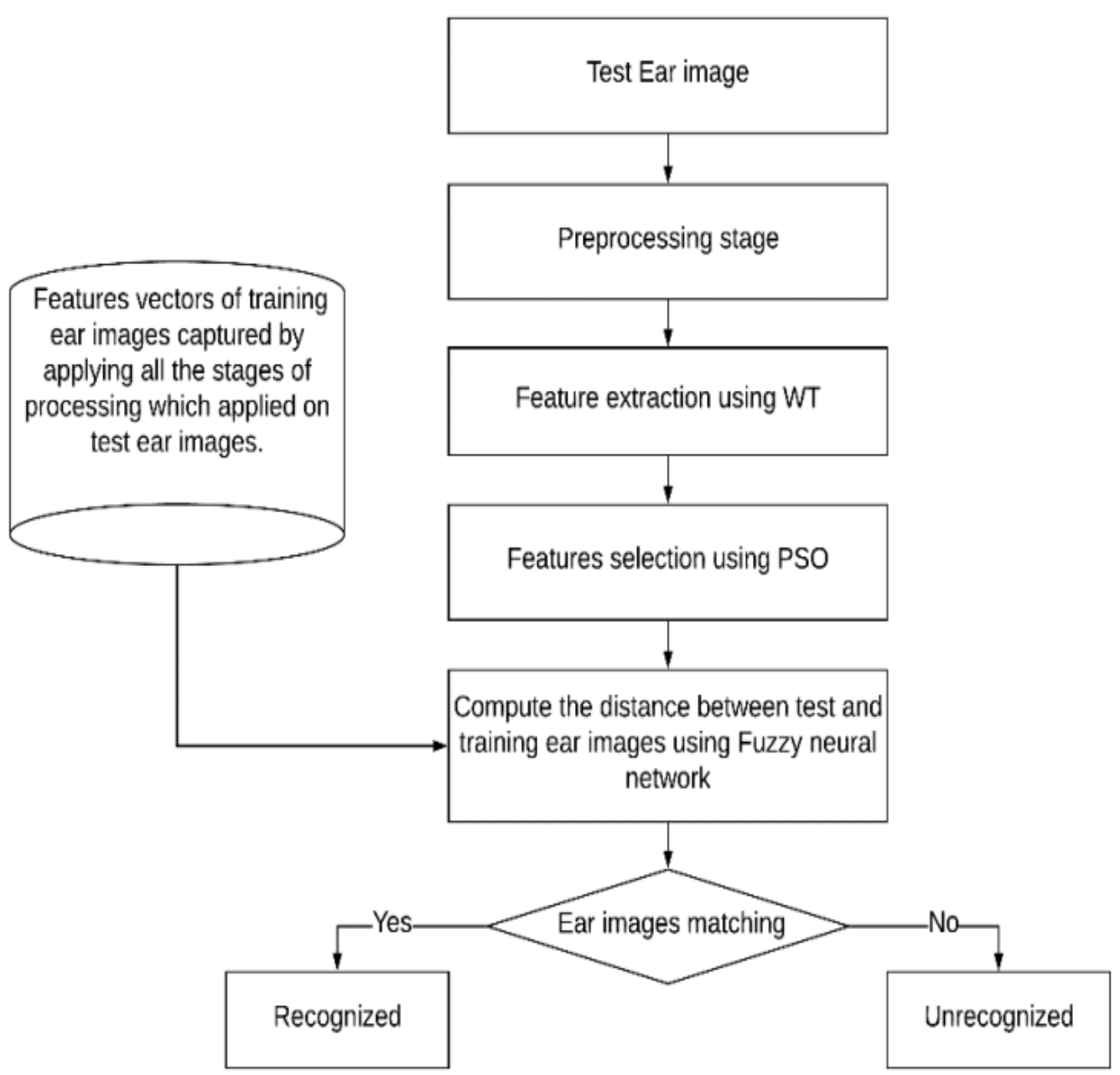

Fig. 1. The proposed technique's block diagram.

\subsection{Preprocessing}

The preprocessing stage is play an important role in ear recognition system because it contains an essential operations such as convert ear image from color to gray scale, ear image enhancement, and ear image segmentation.

\subsubsection{Ear image enhancement}

In order to improve the quality of ear image a hybrid approach of Wiener filter with histogram equalization has been utilized. Wiener filter used to eliminate the noise from the ear image, while histogram equalization used to improve ear image contrasts. Fieger 2 shoes some ear images before and after enhancement. 


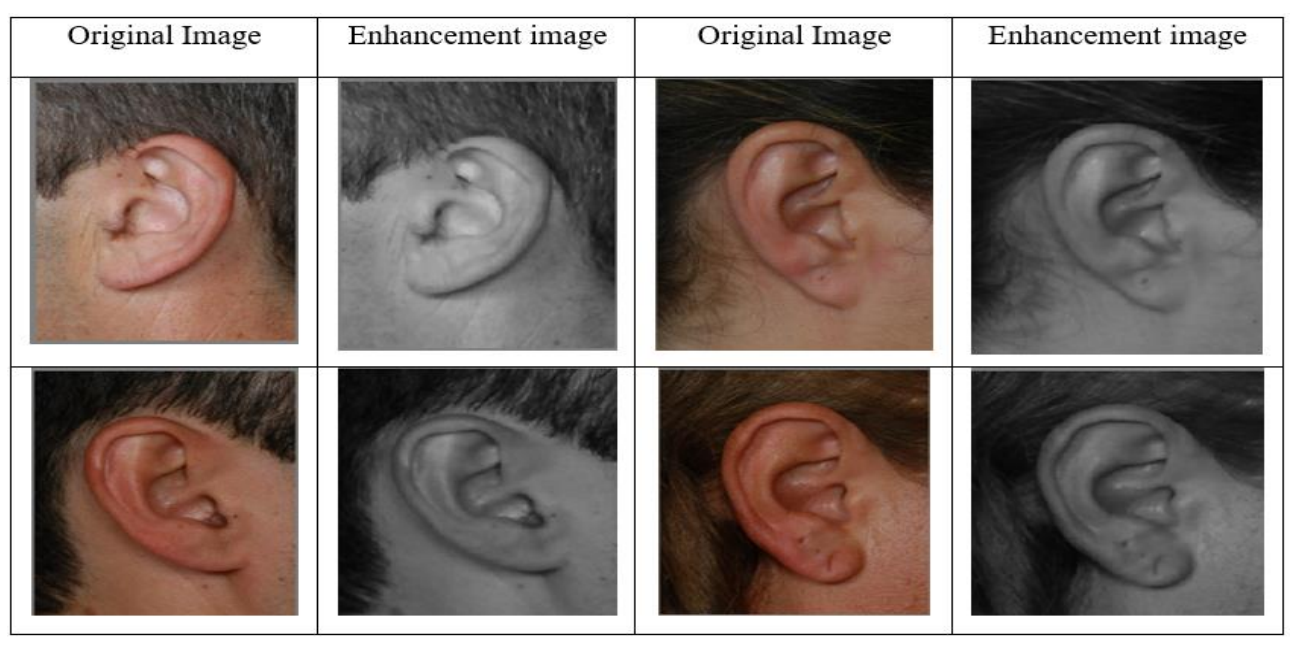

Fig. 2. some ear images before and after enhancement.

\subsubsection{Region of interest segmentation}

Segmentation of an ear image is a technique to partition an image of nonoverlapping regions in order to separate the region of interest (ROI) from unwanted areas such as hair and other details. In this work we have presented a hybrid ear image segmentation based on adaptive mask algorithm and k-means clustering. The proposed ear image segmentation can be explained as follows:

1. Apply adaptive mask algorithm: in this step the best mask for the segmentation is selected by training a selected ear image with all ear image database.

2. Apply K-means algorithm: in this step the k-means algorithm is used with best mask resulted in step 1 as well as the Euclidian distance in order to determine the large objects in ear image. In this work the $\mathrm{k}$ value in $\mathrm{k}$-means algorithm is 3 .

3. Apply morphology operations: the purpose of utilizing morphology operations is to select large shapes and discard the small shapes as well as full the empty regions in ear image, then enlarge the final extracted ROI of ear image. Figure 3 shows some ear image before and after segmentation. 


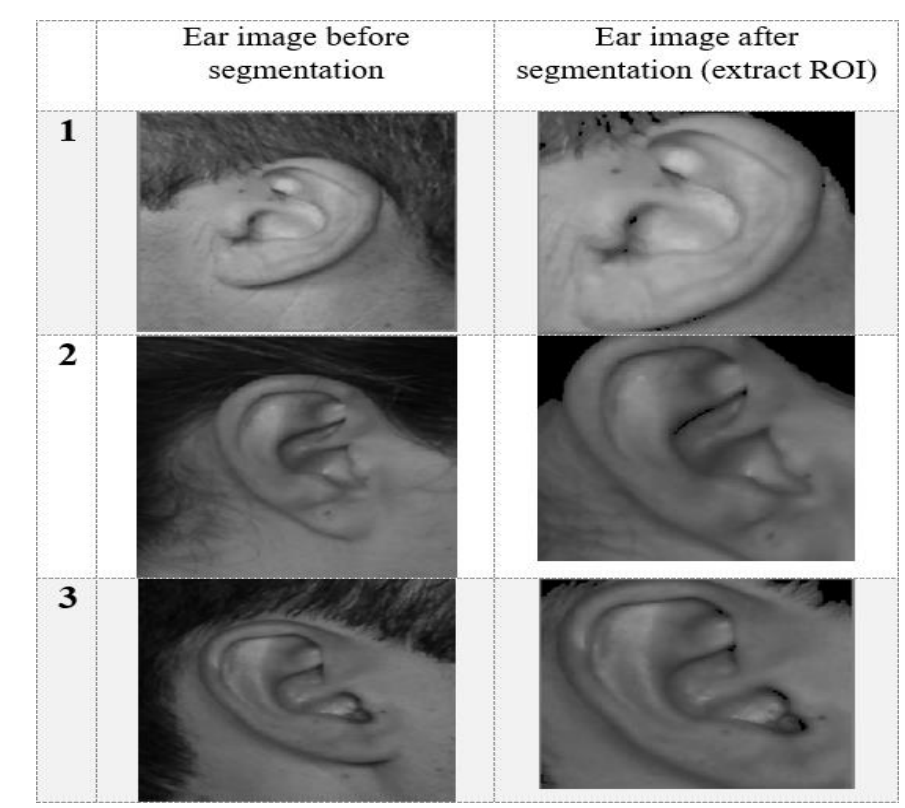

Fig. 3. some ear images before and after segmentation process.

\subsection{Feature extraction}

In this stage the features of the enhanced and segmented testing and training ear images are extracted in order to construct the feature vectors. For this purpose, we used DWT to extract the effective features of the segmented ear images. The DWT provides hierarchically decomposing of an image resulted frequency features at different levels. Apply DWT on 2D image leads to provide four subbands which are one 2D approximation sub-band (LL) and three 2D detail subbands (LH,HL,HH). In order to reduce the dimensionality, we utilized the coefficients of LL sub-band and discarded the coefficients of other sub-bands. In this work the daubches2 (db2) DWT has been considered because of its robustness compared with other wavelets [28, 29].

\subsection{Feature selection}

The PSO is an optimization scheme affirming on search area in order to obtain the best solution over the problem irrespective of the search range. The PSO contains a set of particles which initialized randomly, a potential solution to the problem is providing by each posing. Certain particles possess a stochastic memory, covering their own most beneficial and the best solutions amongst all the possible particles. The best solution is obtained by repeating the evaluation of the resulted solutions using a fitness measure. The PSO concept is based on the social and movement behaviour of fish groups or birds swarm. The birds are flying from one place to another during the searching of the food, some birds in the swarm have the capability to recognize the scent of food effectively. The birds in the swarm are transferring the information of places that contain the food between 
them in order to exploit these places to find most suitable food. All the elements that are proposed solutions are placed in random locations, and they move randomly within the specified scope of research in a direction that changes gradually, to ensure obtaining a better location than the previous one, and to calculate the best site is based on two criteria, namely the speed and the initial location of all the elements, and they are randomly chosen starting from the new speed will be updated according to the following equation [30]:

$$
V i+1=w V i+C 1 R 1(L B i-x i)+C 2 R 2(G B-x i)
$$

Where, $V$ describes the velocity of the element, $i$ is index value , $x$ represents the location of the element, $(R 1, R 2)$ represent independent factors randomly distributed from (0) to (1), C1, C2 represent acceleration constants and $w$ represents the weight of inertia (weight of inertia). LBiand $G B$ refers to local and global best positions respectively.

The new location can be calculated as follows:

$$
x \_(i+1)=x \_i+V_{-}(i+1)
$$

The weight of inertia (w) is used to avoid the high speed of the swarm, because the high velocity of the swarm may lose the universal particles, the optimum move towards an optimal value. The weight of inertia can computed using the following function [31,32].

$$
W^{(t+1)}=W \_ \text {max }-\left(\left(W \_ \text {max }-W \_ \text {min }\right) / T \_ \text {max }\right) t, W \_\max >W \_ \text {min }
$$

Where, $W \_$max represents the maximum limit of the inertia weight value, $W \_$min represents the minimum inertia weight value, $T_{-} \max$ represents the maximum limit of the number of the selected iterations, $t$ is number of iterations specified.

\subsection{Classification stage}

The fuzzy neural network is a hybrid system consist of artificial neural network and fuzzy logic. The goal of these hybrid systems is to exploit the characteristics of both artificial neural network and fuzzy logic that simulate the human perception. They have some disadvantages that almost disappear by combining them together.

The layers of fuzzy neural network can be described as follows [33]:

First layer: - The layer that corresponds to the input where this layer calculates the share of the input set. Each node creates their organic grades for an appropriate blur group using the organic functions, as shown in equations $(4,5)$

$$
\begin{aligned}
& O_{1, i}=\mu A_{i}(x) \text { for } i=1,2 \\
& \mathrm{OR} \\
& O_{1, i}=\mu B_{i}(y) \text { for } i=1,2
\end{aligned}
$$


Where $O_{L, i}$ represents the outputs of all nodes in a layer $L, x, y$ are the inputs of the node to the layer $\mathrm{L}, i$ is the node number, and $A, B$ are the language labels that have the appropriate organic functions $\mu A_{i}$ and $\mu B_{i}$, respectively. The Gaussian and bell-shape organic function is more commonly used to identify blurry groups. As shown in equation (6)

$\mu A(x)=\frac{1}{1+\left|\frac{x-c_{i}}{a_{i}}\right|^{2 b_{i}}}$

Where $\{a, b, c\}$ are the parameters of the organic functions and $i$ is index value. These parameters are referring to as part of a fuzzy hypothesis.

Second layer: - Calculates the strength of the fuzzy bases by rule, here the AND operator is used to obtain one result representing the outputs of those bases, and then the $\mathrm{O}_{2}$ output from this layer is the result of the signal given by the first layer, as shown in equation (7).

$$
O_{2, i}=w_{i}=\mu A_{i}(x) * \mu B_{i}(y), i=1,2
$$

Third layer: in this layer the firing force of each rule is calculated based on all the rules. Outputs are called normalization of strengths, and $\bar{w}$ is considered as a normal firing force. As shown in equation (8).

$$
O_{3, i}=\varpi=\frac{\omega_{i}}{\omega_{1}+\omega_{2}}, i=1,2
$$

Fourth layer: It contains parable nodes. In this layer the node function calculates the contribution of each rule to the total output. As shown in equation (9)

$$
O_{4, i}=\varpi_{i} f_{i}=\varpi_{i}\left(P_{i} X+q_{i} y+r_{i}\right), i=1,2
$$

Where $\bar{W}_{W}$ is the natural launch power of the third layer $(p, q, r)$ are the coefficients of this linear structure and are also the parameter specified in the resulting part of the surgeon fuzzy model.

Fifth layer: Is the last layer that gives the result. This layer contains a fixed and single node called the summation which represent the output. As shown in equation (10)

$$
\text { Overall output }=O_{5,1}=\sum_{i=1}^{4} \varpi_{i} f_{i}=\frac{\sum_{i=1}^{4} \omega_{i} f_{i}}{\sum_{i=1}^{4} \omega_{i}}
$$

\section{Results and Discussion}

The accuracy of the suggested ear recognition approach has been examined by carried out extensive experiments based on two standard ear databases, IIT Delhi and Ami Ear Databases. We have implemented the proposed approach using 
MATLAB- 12 on a computer under Windows environment with i5 CPU and 8GB RAM.

\subsection{The utilized databases.}

The details below describe the used databases:

1. AMI Ear Database I and II [34]: created by Esther Gonzalez. It includes photos of staff, teachers, and students of the University of Las Palmas - computer science department at the in Spain. Seven images in different directions were taken for each person, the accuracy of these images is $492 \times 702$ pixels, and the file type is Joint Photographic experts Group (jpg). All photos were captured using the Nikon D100 camera under the same situation like lighting conditions, distance from the camera. Figure 4 presented some samples of the AMI Ear Database I and II.

2. IIT Delhi ear database [35]: consist of 121 individuals, each one has minimum a three ear images. The age of individuals in this database is $14-58$ years. The database contain 471 ( $272 \times 204$ pixels) images with jpg format. Recently, a larger version of this database is presented for 212 individuals with total number of 754 ear images. Figure 4 shows some samples of the utilized databases.

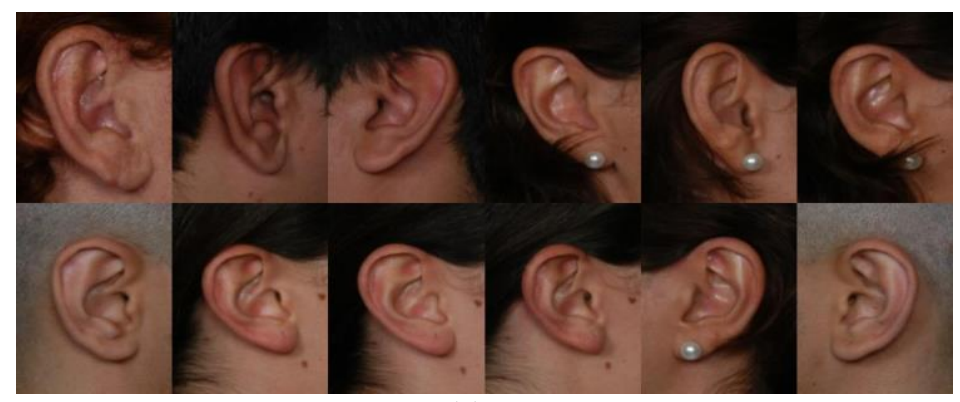

(a)

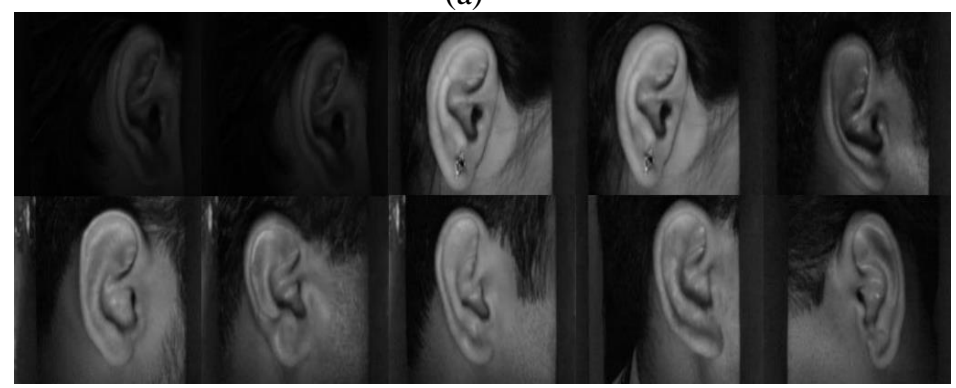

(b)

Fig. 4 some samples of utilized ear databases: (a) some samples of AMI I and II ear database, (b) some samples of IIT Delhi ear database. 


\subsection{Evaluation the proposed technique using AMI ear database1:}

The proposed technique has been evaluated using Ami Ear Database1. For this purpose, we have carried out two experiments. The dataset of first experiment contain 175 images, 150 images for training and 25 images for testing. In the second experiment we enlarged the dataset for both the training and testing therefore, we assigned 50 and 300 images for testing and training respectively. In both experiments the recognition rate calculated for the two levels of DWT and three selected feature sets provided by PSO which are $(8 * 8)$ and $(16 * 16)$ and $(32 * 32)$. The recognition rate (accuracy) is computed as follows:

Recognition Rate (R.R) = (the total no. of the recognized images / The total no. of test images)*100

The tables 1 and 2 presented the results of the first experiment while, table 3 presented the results of the second experiment.

Table 1: Results of the first experiment under the first level of DWT

\begin{tabular}{|c|c|c|c|c|c|}
\hline \multicolumn{2}{|c|}{$\begin{array}{c}\text { DWT (128) \& PSO } \\
(8)\end{array}$} & \multicolumn{2}{c|}{$\begin{array}{c}\text { DWT (128) \& PSO } \\
(16)\end{array}$} & \multicolumn{2}{c|}{ DWT (128) \& PSO (32) } \\
\hline Time(s) & R.R & Time(s) & R.R & Time(s) & R.R \\
& & & & & \\
\hline 26 & $100 \%$ & 29 & $99 \%$ & 30 & $98 \%$ \\
\hline 26 & $100 \%$ & 29 & $100 \%$ & 30 & $98 \%$ \\
\hline
\end{tabular}

Table 2: Results of the first experiment under the second level of DWT

\begin{tabular}{|c|c|c|c|c|c|}
\hline \multirow{2}{*}{ DWT (64) \& PSO (8) } & \multicolumn{2}{|c|}{$\begin{array}{c}\text { DWT (64) \& PSO } \\
(16)\end{array}$} & \multicolumn{2}{|c|}{ DWT (64) \& PSO } \\
(32)
\end{tabular}


Table 3: Results of the second experiment under the second level of DWT

\begin{tabular}{|c|c|c|c|c|c|}
\hline \multicolumn{2}{|c|}{ DWT (64) \& PSO (8) } & \multicolumn{2}{|c|}{ DWT (64) \& PSO (16) } & \multicolumn{2}{|c|}{$\begin{array}{c}\text { DWT (64) \& PSO } \\
\text { (32) }\end{array}$} \\
\hline \multirow{2}{*}{ Time(s) } & R.R & Time(s) & R.R & Time(s) & R.R \\
& & & & & \\
\hline 42 & $98 \%$ & 46 & $98 \%$ & 50 & $97 \%$ \\
\hline 42 & $98 \%$ & 46 & $98 \%$ & 50 & $97 \%$ \\
\hline
\end{tabular}

The results presented in tables 1,2, and 3 refer that the proposed ear recognition technique achieved high recognition rate at the first level of DWT using $8 * 8$ selected features provided by PSO. Furthermore, using PSO leads to decrease the time required for the classification stage as well as using PSO provides high recognition accuracy with less number of features.

\subsection{Evaluate the proposed technique using IIT Delhi Ear Database:}

The accuracy of the suggested technique was investigated on the IIT Delhi ear database. One ear image is randomly selected testing while, remaining considered for training therefore, the total number of training ear images is 90 while, the total number of testing ear images is 15 . In this experiment we repeat the randomly selection test image two times. As in previous experiments we calculate the recognition accuracy for the first and second levels of DWT, and we have considered the same size of selected features sets provided by PSO in the previous experiments. The tables 4 and 5 presented the results of first and second level of DWT respectively.

Table 4: The accuracy results for the first level DWT and PSO are equal to $8 * 8$, $16 * 16$ and $32 * 32$ on IIT Delhi ear database.

\begin{tabular}{|c|c|c|c|c|c|c|}
\hline \multirow{2}{*}{$\begin{array}{c}\text { The randomly } \\
\text { selected test } \\
\text { images }\end{array}$} & \multicolumn{2}{|c|}{$\begin{array}{c}\text { DWT (128) \& } \\
\text { PSO (8) }\end{array}$} & $\begin{array}{r}\text { DWT (128) \& PSO } \\
(16)\end{array}$ & \multicolumn{2}{|c|}{$\begin{array}{c}\text { DWT (128) \& } \\
\text { PSO (32) }\end{array}$} \\
\cline { 2 - 7 } & Time(s) & R.R & Time(s) & R.R & Time(s) & R.R \\
\hline $\begin{array}{c}\text { First randomly } \\
\text { selection test } \\
(15) \text { ear images }\end{array}$ & 18 & $99 \%$ & 19 & $99 \%$ & 20 & $\% 98$ \\
\hline $\begin{array}{c}\text { Second } \\
\text { randomly } \\
\text { selection test } \\
(15) \text { ear images }\end{array}$ & 18 & $100 \%$ & 19 & $100 \%$ & 20 & $100 \%$ \\
\hline
\end{tabular}


Table 5: The accuracy results for the second level of DWT and PSO are equal to 8 $* 8,16 * 16$ and $32 * 32$ on IIT Delhi ear database.

\begin{tabular}{|c|c|c|c|c|c|c|}
\hline \multirow{2}{*}{$\begin{array}{c}\text { The } \\
\text { randomly } \\
\text { selected } \\
\text { test images }\end{array}$} & \multicolumn{2}{|c|}{ DWT (64) \& PSO (8) } & \multicolumn{2}{|c|}{$\begin{array}{c}\text { DWT (64) \& PSO } \\
\text { (16) }\end{array}$} & \multicolumn{2}{|c|}{$\begin{array}{c}\text { DWT (64) \& PSO } \\
\text { (32) }\end{array}$} \\
\cline { 2 - 7 } & Time(s) & R.R & Time(s) & R.R & Time(s) & R.R \\
\hline $\begin{array}{c}\text { First } \\
\text { randomly } \\
\text { selection } \\
\text { test (15) ear } \\
\text { images }\end{array}$ & 15 & $99 \%$ & 16 & $99 \%$ & 18 & $98 \%$ \\
\hline $\begin{array}{c}\text { Second } \\
\text { randomly } \\
\text { selection } \\
\text { test (15) ear } \\
\text { images }\end{array}$ & 15 & $100 \%$ & 16 & $100 \%$ & 18 & $100 \%$ \\
\hline
\end{tabular}

From the results presented in 4 and 5 tables, it observed that the proposed system gives a high accuracy up to $100 \%$ with IIT Delhi ear database with decrees the time taken for the classification from $20 \mathrm{~s}$ into $18 \mathrm{~s}$ for first level of DWT, while in the second level of DWT the time is reduced from $18 \mathrm{~s}$ into $15 \mathrm{~s}$ with accuracy of $99 \%$. This is because of that using DWT \& PSO leads to decrease the dimensionality of these features and consequence decreases the complexity of the classification stage. In all above experiments, it can be observed that the time taken using second level of DWT is less than the time taken using first DWT because that the dimensionality of ear images in first level of DWT is larger than their dimensionality in second level of DWT

\section{4 comparing the proposed method with some recent methods}

We compare the proposed method with the accuracy of some other recent presented methods using the same ear databases utilized in these methods. Table 6 presented the results of this comparison. These results refer that proposed technique outperform the compared methods, as well as it utilized techniques such as DWT \& PSO to improve the efficiency of the proposed work. 
Table 6: the results of the comparison between the proposed method and some recent method

\begin{tabular}{|c|c|}
\hline Method & R.R \\
\hline LBP/Laplacian filter method [36] & $80 \%$ \\
\hline Deep earprint learning [37] & $94 \%$ \\
\hline The method in [38] & $97.36 \%$ \\
\hline Proposed method & $99 \%$ \\
\hline
\end{tabular}

\section{Conclusions}

The experimental analysis indicated the following conclusions:

1. The proposed ear recognition technique achieves high recognition rates using different ear datasets.

2. the complexity of the proposed method has been reduced two times, the first one by using DWT and considered the LL band only, while the second one is achieved by using the PSO algorithm in order to select an active subset feature.

3. Using PSO algorithm leads also to increase the recognition accuracy of the proposed technique.

4. Using FNN in the classification stage leads to achieves high classification accuracy compared with the traditional distance measure like Euclidian distance.

\section{References}

[1] J. Xiong, Essential bioinformatics. Cambridge University Press, 2006.

[2] Ali Sami Azeez, "Rotation Invariant Face Recognition Using Radial Harmonic Fourier Moments," J. Coll. basic Educ., vol. 23, no. 99, pp. 8798, 2017.

[3] K. Rabuzin, M. Baca, and M. Sajko, "E-learning: Biometrics as a Security Factor," in 2006 International Multi-Conference on Computing in the Global Information Technology-(ICCGI'06), 2006, p. 64.

[4] W. Yang, S. Wang, J. Hu, G. Zheng, and C. Valli, "Security and accuracy of fingerprint-based biometrics: A review," Symmetry (Basel)., vol. 11, no. 2, p. 141, 2019.

[5] K. Revett, Behavioral biometrics: a remote access approach. John Wiley \& Sons, 2008.

[6] S. Marcel, M. S. Nixon, and S. Z. Li, Handbook of biometric antispoofing, vol. 1. Springer, 2014. 
[7] F. Monrose and A. D. Rubin, "Keystroke dynamics as a biometric for authentication," Futur. Gener. Comput. Syst., vol. 16, no. 4, pp. 351-359, 2000.

[8] L. O'Gorman, "Comparing passwords, tokens, and biometrics for user authentication," Proc. IEEE, vol. 91, no. 12, pp. 2021-2040, 2003.

[9] L. O'Gorman, “Securing Business's Front Door-Password, Token, and Biometric Authentication."

[10] P. Punithavathi and S. Geetha, "Partial DCT-based cancelable biometric authentication with security and privacy preservation for IoT applications," Multimed. Tools Appl., pp. 1-28, 2019.

[11] H. Senses, "The Human Ear-Hearing, Sound Intensity and Loudness Levels," 2013.

[12] E. P. Widmaier, H. Raff, K. T. Strang, and A. J. Vander, Vander's Human physiology: the mechanisms of body function., Fifteenth. New York: McGraw-Hill Higher Education, 2019.

[13] V. Murgod, P. Angadi, S. Hallikerimath, and A. Kale, "Anthropometric study of the external ear and its applicability in sex identification: assessed in an Indian sample," Aust. J. Forensic Sci., vol. 45, no. 4, pp. 431-444, 2013.

[14] B. Bhanu and H. Chen, Human ear recognition by computer. Springer Science \& Business Media, 2008.

[15] S. Singh and S. V. A. V. Prasad, "Techniques and Challenges of Face Recognition: A Critical Review," Procedia Comput. Sci., vol. 143, pp. 536-543, Jan. 2018.

[16] M. Choraś, "Intelligent computing for automated biometrics, criminal and forensic applications," in International Conference on Intelligent Computing, 2007, pp. 1170-1181.

[17] L. Nanni and A. Lumini, "Fusion of color spaces for ear authentication," Pattern Recognit., vol. 42, no. 9, pp. 1906-1913, 2009.

[18] L. Feenstra and C. Van der Lugt, "Ear witness," J. Laryngol. Otol., vol. 114, no. 7, pp. 497-500, 2000.

[19] L. Meijerman, C. Van Der Lugt, and G. J. R. Maat, "Cross- sectional anthropometric study of the external ear," J. Forensic Sci., vol. 52, no. 2, pp. 286-293, 2007.

[20] A. J. Hoogstrate, H. Van Den Heuvel, and E. Huyben, "Ear identification based on surveillance camera images," Sci. Justice, vol. 41, no. 3, pp. 167$172,2001$.

[21] J. C. Klontz and A. K. Jain, "A case study on unconstrained facial recognition using the boston marathon bombings suspects," Michigan State Univ. Tech. Rep, vol. 119, no. 120, p. 1, 2013.

[22] P. Yan, Ear biometrics in human identification. University of Notre Dame, 2006. 
[23] V. K. N. Kumar and B. Srinivasan, "Ear biometrics in human identification system," Int. J. Inf. Technol. Comput. Sci., vol. 4, no. 2, pp. 41-47, 2012.

[24] A. S. Anwar, K. K. A. Ghany, and H. Elmahdy, "Human Ear Recognition Using Geometrical Features Extraction,” Procedia Comput. Sci., vol. 65, pp. 529-537, Jan. 2015.

[25] C. Cintas et al., "Automatic ear detection and feature extraction using geometric morphometrics and convolutional neural networks," IET Biometrics, vol. 6, no. 3, pp. 211-223, 2016.

[26] I. Omara, X. Wu, H. Zhang, Y. Du, and W. Zuo, "Learning pairwise SVM on hierarchical deep features for ear recognition," IET Biometrics, vol. 7, no. 6 , pp. 557-566, 2018.

[27] A. A. A. Al-Dulaimi, J. M. A. Al-Tuwaijari, and Z. Mustafa Al-Ta'i, "Human ear recognition using surf-Pso technique," J. Theor. Appl. Inf. Technol., vol. 97, pp. 825-834, Feb. 2019.

[28] P. Gonzalo, J. M. De La Cruz, "A wavelet-based image fusion tutorial", Pattern Recognition, 2004, Vol. 37, pp. 1855 - 1872.

[29] H. Ma, C. Jia and S. Liu, "Multisource Image Fusion Based on Wavelet Trans form", Int. Journal of Information Technology, Vol. 11, No. 7, 2005.

[30] Y. Chen, L. Li, H. Peng, J. Xiao, Y. Yang, Y. Shi, "Particle swarm optimizer with two differential mutation", Applied Soft Computing, vol. 61, pp. 314-330, 2017.

[31] R. Eberhart, J. Kennedy, "A new optimizer using particle swarm theory," in Micro Machine and Human Science, 1995. MHS'9 ,.5Proceedings of the Sixth International Symposium on, pp. 39-43,1995.

[32] Y. Shi, R. Eberhart, "A modified particle swarm optimizer," in Evolutionary Computation Proceedings, IEEE World Congress on Computational Intelligence., pp. 69-73,1998.

[33] O. E. Dragomir, F. Dragomir, V. Stefan, E. Minca," Adaptive NeuroFuzzy Inference Systems as a Strategy for Predicting and Controling the Energy Produced From Renewable Sources", Energies, 8, 1304713061, 2015.

[34] http;//ctim.ulpgc.es/research_works/ami_ear_database

[35] http://www4.comp.polyu.edu.hk/ csajaykr/IITD/Database_Ear.htm

[36] S. M. Jiddah, K. Yurtkan,"Fusion of Geometric and Texture Features for Ear Recognition", 2nd International Symposium on Multidisciplinary Studies and Innovative Technologies (ISMSIT), October 2018.

[37] A. H. Salih Hamdany, A. Thamar Ebrahem, A. M. Alkababji“", Earprint Recognition using deep learning technique", TELKOMNIKA elecommunication, Computing, Electronics and Control), vol. 19, no. 2, pp. 432-437, 2021

[38] R. Ahila Priyadharshini, S. Arivazhagan, M. Arun, "A deep learning approach for person identification using ear biometrics", Applied intelligence (2021) 51: 2161-2172. 


\section{Notes on contributors}

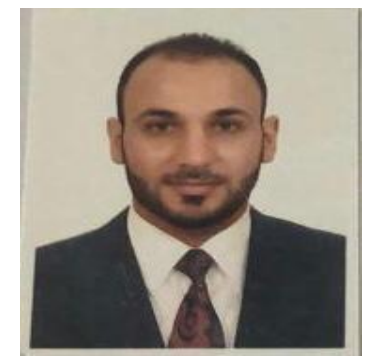

Yahya Abdulmunem Hussein $\mathrm{He}$ has done his M.Sc. informatics in 2020. His research interests include Digital Image processing, Pattern Recognition and Artificial Intelligent.

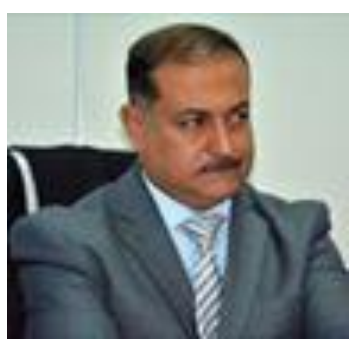

Dr. ALI Mohammed Sahan is associate Professor at the Department of Informatics, Meddle Technical University, Iraq, Baghdad. His research interests include Digital Image processing, Pattern Recognition and Artificial Intelligent. He has published several research articles in international journals of computer science, as well as he is reviewer and editor in several international journals.

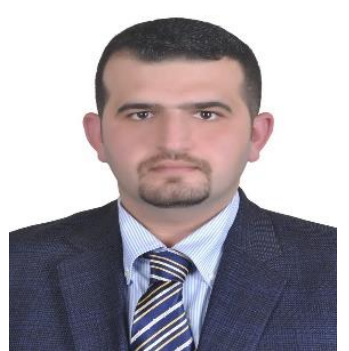

Ali Sami Al-Itbi is Lecturer at the Department of Informatics, Meddle Technical University, Iraq, Baghdad. He has done his M.Sc. Computer science in 2014. His research interests include Digital Image processing, Pattern Recognition and Artificial Intelligent. He has published several research articles in international journals of computer science 